\title{
Design and analysis of an $x$-ray mirror assembly using the meta-shell approach
}

\author{
Ryan S. McClelland*a, Joseph A. Bonafede ${ }^{\mathrm{a}}$, Timo T. Saha ${ }^{\mathrm{b}}$, Peter M. Solly ${ }^{\mathrm{a}}$ William W. Zhang ${ }^{\mathrm{b}}$ \\ ${ }^{a}$ SGT Inc. 7701 Greenbelt Road, Suite 400, Greenbelt, Maryland 20770, USA \\ bNASA Goddard Space Flight Center (GSFC), Greenbelt, MD USA 20771, USA
}

\begin{abstract}
Lightweight and high resolution optics are needed for future space-based x-ray telescopes to achieve advances in highenergy astrophysics. Past missions such as Chandra and XMM-Newton have achieved excellent angular resolution using a full shell mirror approach. Other missions such as Suzaku and NuSTAR have achieved lightweight mirrors using a segmented approach. This paper describes a new approach, called meta-shells, which combines the fabrication advantages of segmented optics with the alignment advantages of full shell optics. Meta-shells are built by layering overlapping mirror segments onto a central structural shell. The resulting optic has the stiffness and rotational symmetry of a full shell, but with an order of magnitude greater collecting area. Several meta-shells so constructed can be integrated into a large x-ray mirror assembly by proven methods used for Chandra and XMM-Newton.

The mirror segments are mounted to the meta-shell using a novel four point semi-kinematic mount. The four point mount deterministically locates the segment in its most performance sensitive degrees of freedom. Extensive analysis has been performed to demonstrate the feasibility of the four point mount and meta-shell approach. A mathematical model of a meta-shell constructed with mirror segments bonded at four points and subject to launch loads has been developed to determine the optimal design parameters, namely bond size, mirror segment span, and number of layers per meta-shell. The parameters of an example $1.3 \mathrm{~m}$ diameter mirror assembly are given including the predicted effective area. To verify the mathematical model and support opto-mechanical analysis, a detailed finite element model of a meta-shell was created. Finite element analysis predicts low gravity distortion and low sensitivity to thermal gradients.
\end{abstract}

Keywords: silicon mirrors, meta-shell, module, Mirror Assembly, NGXO, x-ray optics

\section{INTRODUCTION}

Advancements in x-ray optics fabrication technologies are required to enable future discoveries by space-based $\mathrm{x}$-ray telescopes [1]. While both lightweight and high resolution mirror fabrication technologies exist, no spaceflight proven technology currently achieves both at once. Lightweight and relatively low cost mirror assemblies have been constructed for both the Suzaku and NuSTAR missions, with relatively low resolution of 110 arc-seconds HPD and 60 arc-seconds HPD respectively. The low mass of the optics allows for large photon collecting area at the expense of focusing performance. Mission such as Chandra and XMM-Newton have achieved superior angular resolutions of 0.5 arc-seconds HPD and 15 arc-seconds HPD respectively with relatively small collecting area and at a very high cost. Suzaku and NuSTAR used thin segmented mirrors to achieve their low mass, while Chandra and XMM-Newton used relatively thick full shell optics to achieve their excellent resolution. A hybrid approach, called meta-shells, that combines the benefits of both lightweight segmented optics and stiff full shell optics is being pursued by the Next Generation X-ray Optics (NGXO) team at NASA GSFC.

*ryan.s.mcclelland@ nasa.gov; phone 1301 286-8615 


\section{META-SHELL APPROACH TO MODULAR X-RAY OPTICS}

\subsection{Full shell versus segmented optics}

The basic optical elements of current x-ray mirrors can be divided into two types: full shell mirrors, such as those used on ROSAT, Chandra, and XMM-Newton, and segmented mirrors such as those used for Suzaku and NuSTAR. While full shell optics have advantages in stiffness, due to the full annulus, and integration, due to symmetry and reduced number of optics to integrate, they are not amenable to making large lightweight mirror assemblies. As optics increase in collecting area they must also decrease in mass and per unit area to accommodate existing launch vehicles. However, as they decrease in mass, optics become thinner, more flexible, and therefore more easily distorted, particularly during fabrication and mounting. Thus far, full shell optics with a diameter greater than $1 \mathrm{~m}$ and a thickness less than $1 \mathrm{~mm}$ have not been successfully fabricated. For example, Chandra's largest shell was $1.2 \mathrm{~m}$ in diameter with a thickness of 23 $\mathrm{mm}$ [2]. For this reason, the majority of $\mathrm{x}$-ray mirror technology development to enable future large collecting area missions such as Athena and X-Ray Surveyor focuses on thin segmented mirrors [3].
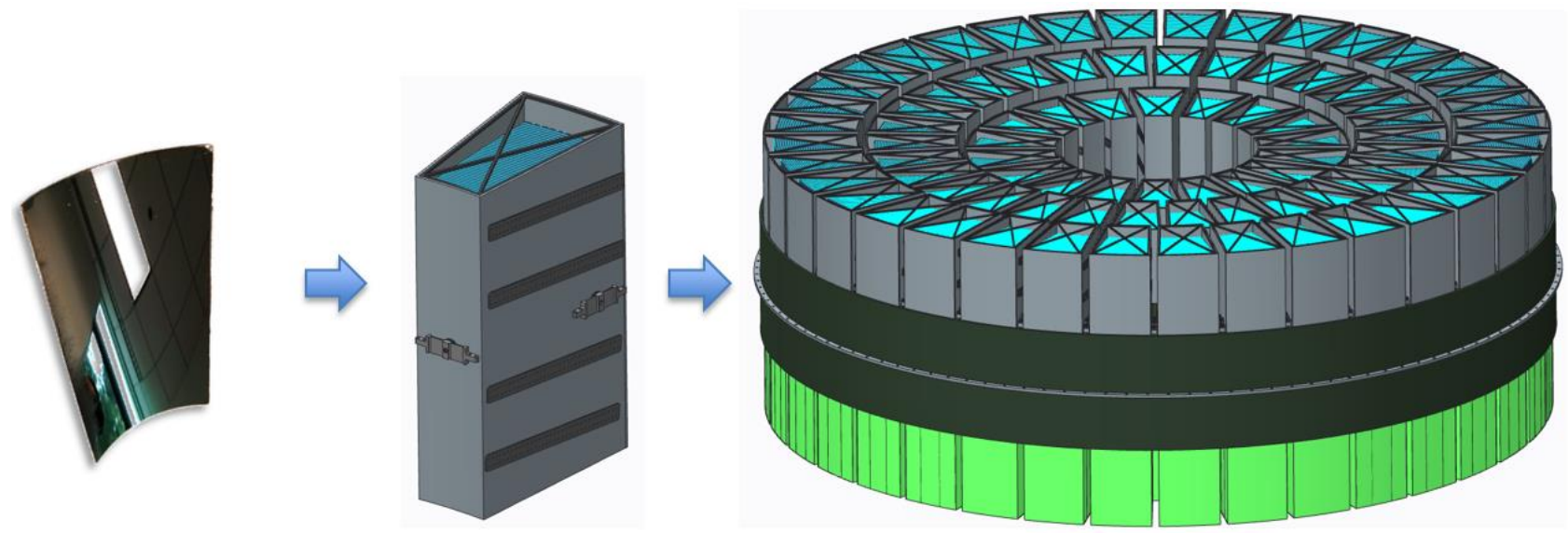

Figure 1. Mirror assembly constructed from wedge-shaped modules.

\subsection{Wedge shaped modules versus meta-shells}

Large mirror assemblies envisioned for future missions have typically been divided up both radially and azimuthally into many wedge shaped modules [4,5] as shown in Figure 1. This paradigm has the advantage of requiring many identical modules of modest size. However, it has distinct disadvantages. First, it sacrifices the rotational symmetry inherent in full shell optics leading to difficulty in establish an optical axis and high sensitivity to roll misalignment (about the modules center of gravity) during module integration. Second, mounting to the module structure leads to over-constraint of the mirror segment which generates mounting distortion.

The meta-shell approach divides the mirror assembly up radially, similar to Chandra, but with each radial division containing dozens of optical shells rather than a single shell. Each radial element, or meta-shell, consists of a thick structural shell (non-optical) onto which many thin shells are layered in an interlocking pattern as shown in Figure 2. This paradigm has the advantage of creating a lightweight and stiff optic with a large collecting area. The meta-shell approach is similar to the construction of the NuSTAR mirrors [6] though it is designed to be scalable for larger mirror assemblies and differs in the particulars of the mirror segment mounting as described in Section 2.3 below. Additionally, the meta-shell is rotationally symmetric which simplifies aligning several meta-shells into a mirror assembly. The same approaches used to align and mount full shell optics for Chandra and XMM-Newton can be used with meta-shells. Finally, the continuous annular structure simplifies thermal design by allowing a conduction path through the structural shell as opposed to wedge-shaped modules which must be individually thermally controlled. 

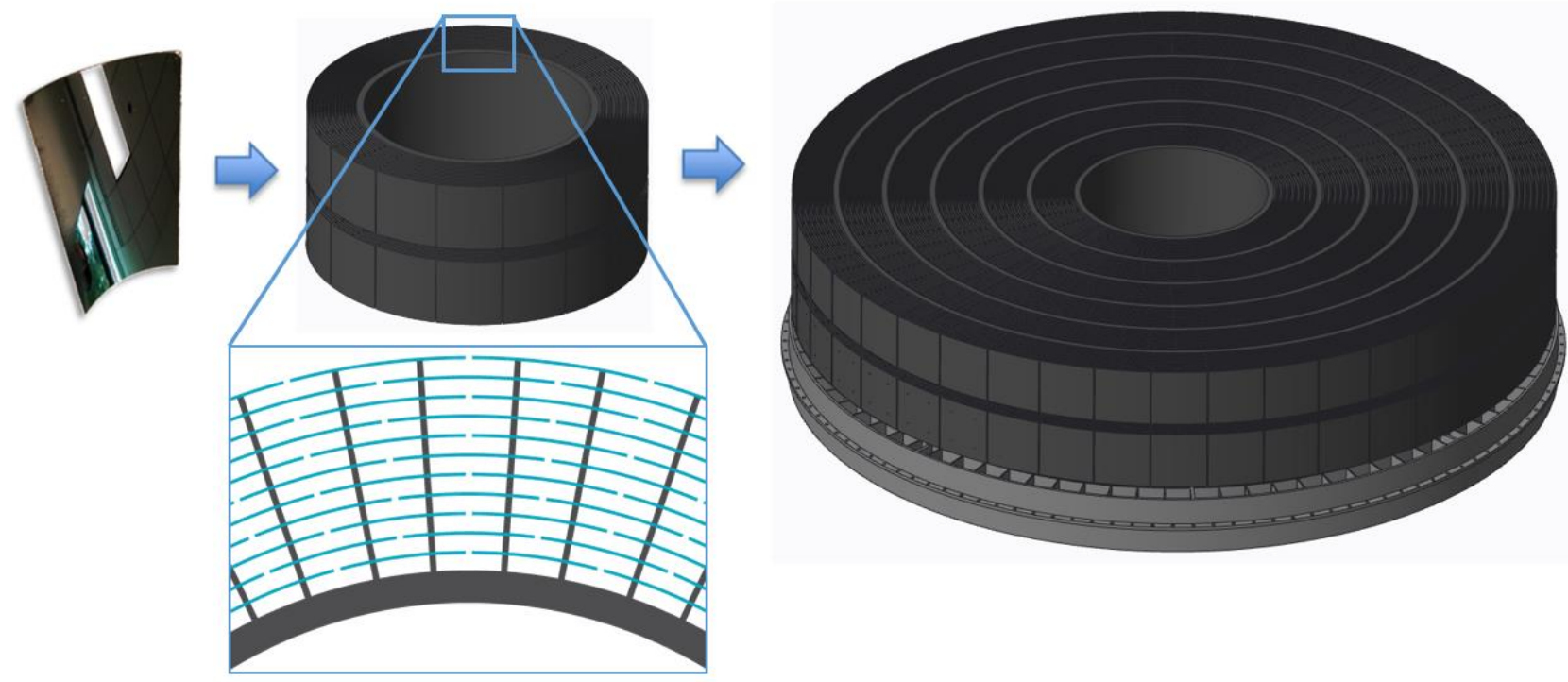

Figure 2. Mirror assembly constructed from meta-shells. Mirror segments are layered onto the structural shell in an interlocking pattern to form the meta-shell. Several meta-shells can be co-aligned to create a large mirror assembly.

\subsection{Four post kinematic mount}

To minimize mirror distortion, a kinematic mount is desired. With a kinematic mount, the stress state is deterministic and the distortion due to gravity can be easily calculated and optimized using Finite Element Analysis (FEA). In comparison, an over-constrained mount can distort a thin mirror segment in ways that are difficult to predict and measure, since the sensitivity to displacement of over-constrained mounts can be as high as 35 arc-seconds HPD / $\mu \mathrm{m}$ [7]. To this end, a kinematic mount using four posts of a prescribed height has been developed.

As three points describe the location and orientation of a plane, so four points describe the location and orientation of a cylinder or cone. A mirror segment mounted on four points has its four most crucial degrees of freedom (dof) constrained, namely de-center ( 2 dof), pitch, and yaw. The remaining two degrees of freedom are roll, which has no effect on the focus quality due to rotational symmetry, and de-space, which is orders of magnitude less sensitive. Once the mirror is so located, it can be bonded in place to a CTE matched structure (silicon in this case) with minimal distortion as shown in Figure 3. Axially, the mounts are located at the Airy points to minimize axial distortion, and azimuthally the mounts are located at the $1 / 4$ points to allow for staggered mounting of the subsequent layer of mirror segments as shown in Figure 2.

Gravity, which provides the nesting force needed to ensure the mirror contacts all four points, distorts the mirror by a predictable amount. The distortion of a $300 \mathrm{~mm}$ diameter silicon mirror segment with gravity acting radially, as during mounting to the meta-shell, is 2.2 arc-seconds HPD. If the mounted mirror is then turned 90 degrees, such that gravity acts azimuthally, the distortion reduces to 0.5 arc-seconds, allowing the mirror to be tested in a horizontal x-ray beam line. With gravity completely absent on orbit, only 0.2 arc-seconds of distortion, frozen in during the bonding process, remain. The minimal distortion of the four post mount has been verified experimentally as shown in Figure 3 . 

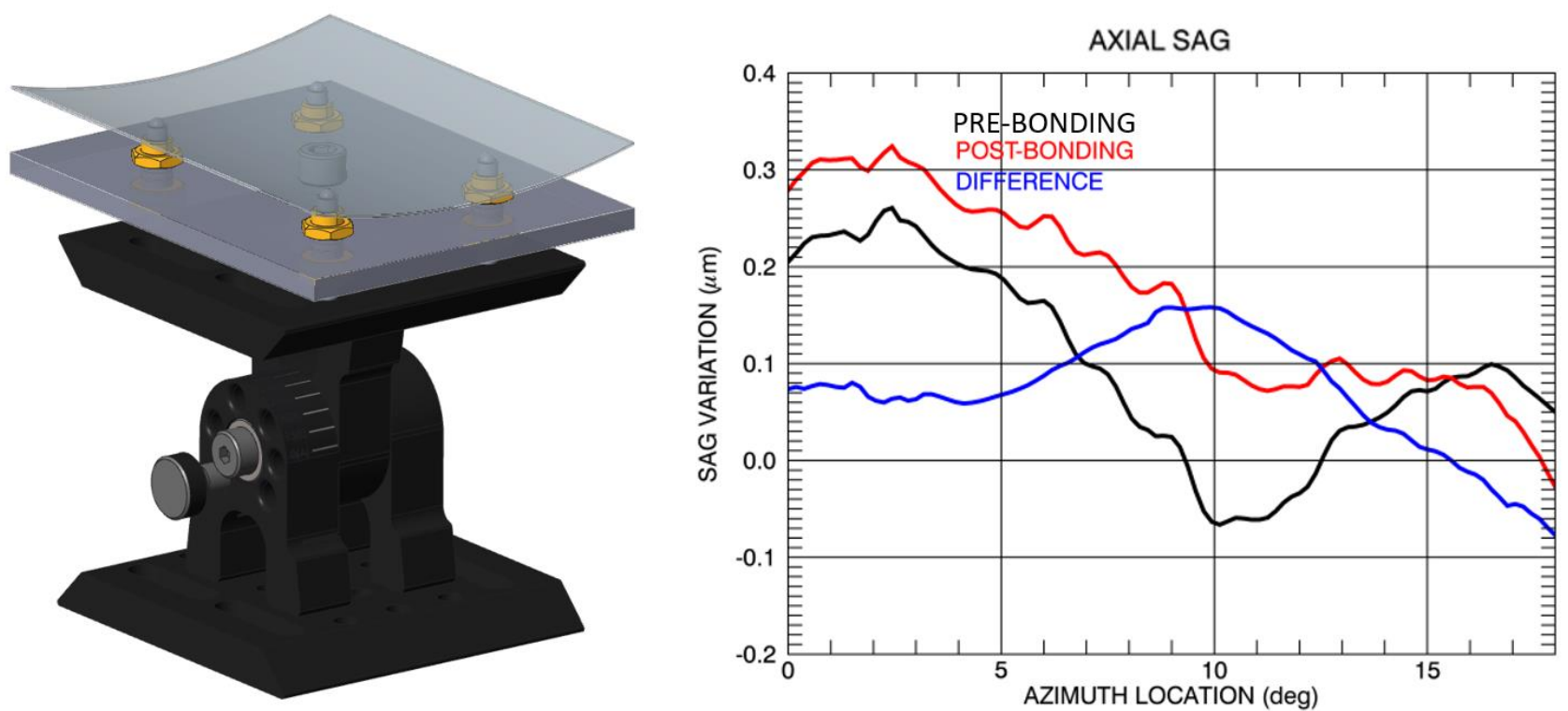

Figure 3. Four point mount bonding development platform (left). The mirror is bonded at four posts, then rotated 90 degrees for interferometric measurement. The change in mirror figure (blue line) is within the metrology system repeatability of $0.15 \mu \mathrm{m}$.

\subsection{Constructing a meta-shell}

Constructing a meta-shell involves five major steps. First, the structural shell is fitted with temporary end-caps and mounted to an air bearing spindle as shown in Figure 4. The axis of rotation defines the optical axis of the meta-shell. Second, posts are bonded to the structural shell to provide the mounting points for the first layer of mirror segments. To simplify alignment, both primary and secondary mirrors are mounted to the same structural shell. Third, the post heights are set using in-situ machining to the required tolerance. The post heights are verified by installing a mirror segment and checking its focus. Fourth, a layer of mirror segments are bonded on top of the posts. Steps two, three, and four are repeated for each layer of mirror segments. The number of mirror segments in a meta-shell is determined by the optical design and bond strength as described in Section 3 below. Finally, the temporary end caps are removed and the metashell is mounted to a Ground Support Equipment (GSE) structure for transportation, testing, and alignment into the mirror assembly.
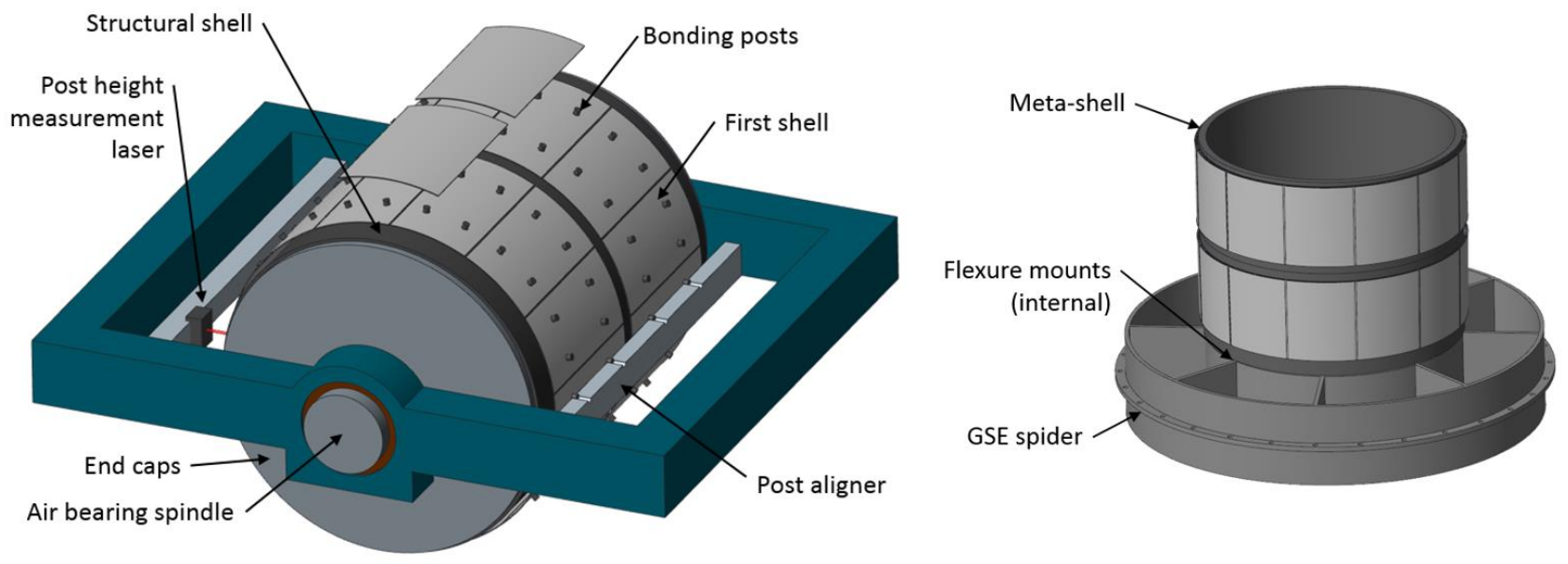

Figure 4. Meta-shells are assembled in on air-bearing spindle (left). After assembly they are mounted to a GSE spider structure for transportation, testing, and alignment (right). 


\subsection{Meta-shell mirror assembly}

Several meta-shells can me aligned and bonded onto a carrier structure to form a mirror assembly. Proven techniques from Chandra and XMM-Newton have been adapted. Similar to XMM-Newton, the meta-shells, which combine primary and secondary mirrors, are mounted at the forward (primary mirror) end to a carrier structural with thin radial spokes called the spider [8] as shown in Figure 5. During integration they are supported by the GSE structure at the secondary mirror end where they can be manipulated for alignment. As with Chandra, alignment can be checked with a steerable auto-collimated beam at the focus which is retro-reflected off a flat mirror at the aperture [9]. Once aligned, the meta-shell is bonded to flexures allowing a radial degree of freedom, again following the Chandra approach [2].

The spider structure, being at the space-facing end of the mirror, can be used to mount stray-light baffles and can also be heated to provide a warm surface to radiate to the mirror segments. The completed mirror assembly includes the spider with mounted meta-shells, the interface ring to provide mounting for the telescope tube and spacecraft, and a thermal pre-collimator as shown in Figure 5. The thermal design is also adapted from Chandra and previous x-ray mission studies [10].

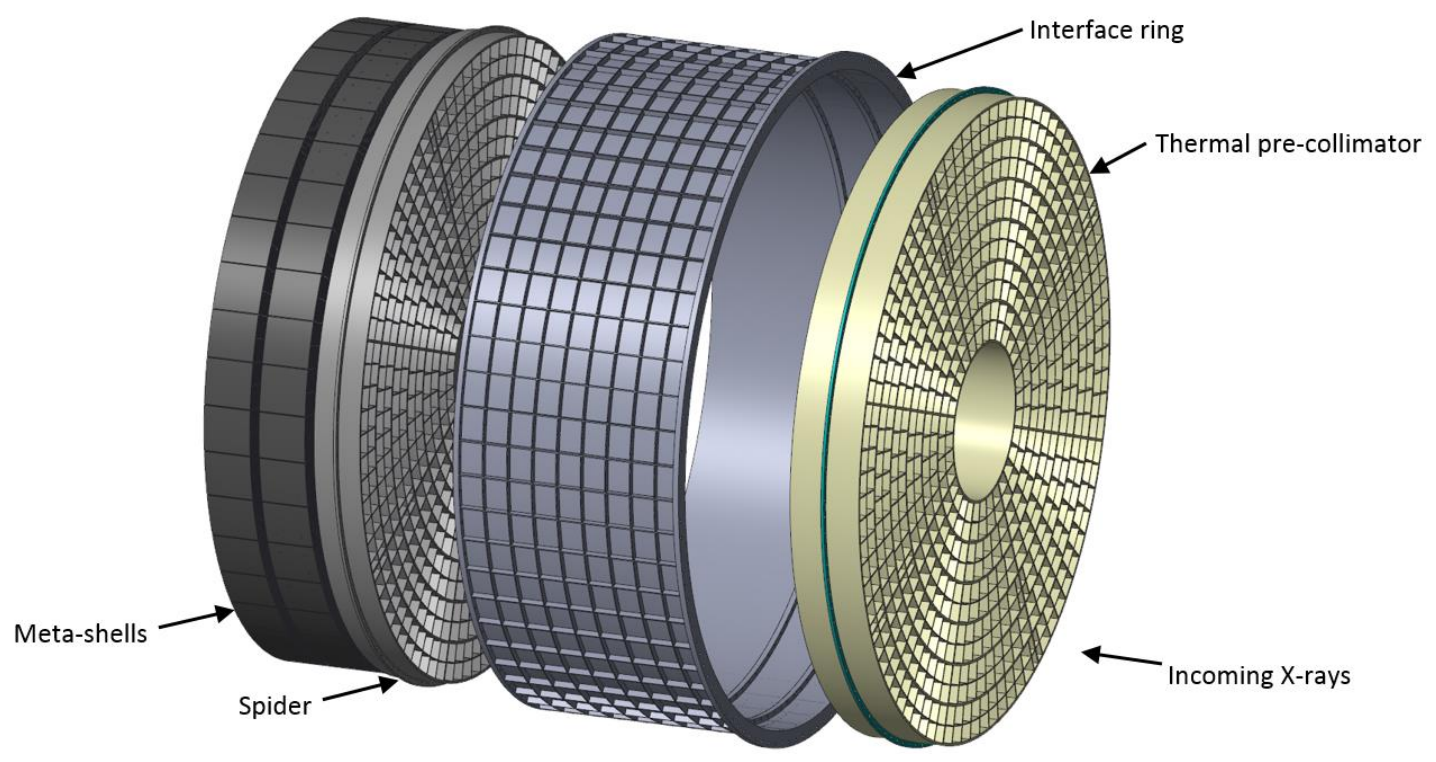

Figure 5. The meta-shells are mounted to a spider structure. The spider structure has additional heated vanes for thermal control. Both the spider structure and thermal pre-collimator are mounted to an interface ring that provides mounting interfaces to the spacecraft and telescope tube.

\section{META-SHELL STRUCTURAL ANALYSIS}

To verify the feasibility of the meta-shell approach and to optimally size the meta-shell components, structural and optomechanical analysis has been performed to check the launch stress in the assembly, the distortion due to gravity, and distortion due to thermal gradients

\subsection{Bond strength versus bond size, mirror span, and number of layers}

With hundreds of thin optical elements bonded onto the structural shell, the strength of the meta-shell when subject to launch loads is an obvious concern. Analysis shows that the weakest point in the design is the bond stress in the adhesive between the structural shell and the innermost mounting post where the load from all the subsequent cantilevered layers of mirrors must be transferred. A mathematical model which calculates the adhesive stress at the bond joints was developed. The model calculates the shear, tensile, and bending stresses in the bond joint and finds the margin of safety based on a conservative safety factor of 3.0. Launch loads of $12.3 \mathrm{~g}$ axial and $3.4 \mathrm{~g}$ axial were applied based on the International X-Ray Observatory (IXO) mission study coupled loads analysis with a model uncertainty factor of 2.0 applied to the loads [7]. The meta-shell design parameters effecting the bond stress are (1) the diameter of the bond between the mirror segment / structural shell and mounting post (2) the azimuthal span of the mirror segment, 
which determines the number of bonds around the meta-shell circumference and (3) the number of mirror layers on the meta-shell, which determines how much load must be carried by the inner-most bonds. Based on these three parameters, the mathematical model was iterated to determine the feasible design region shown in Figure 6. Designs with parameters below the surface shown are feasible, while those above the surface have a negative margin of safety.

\section{0mm Diameter Meta-Shell}
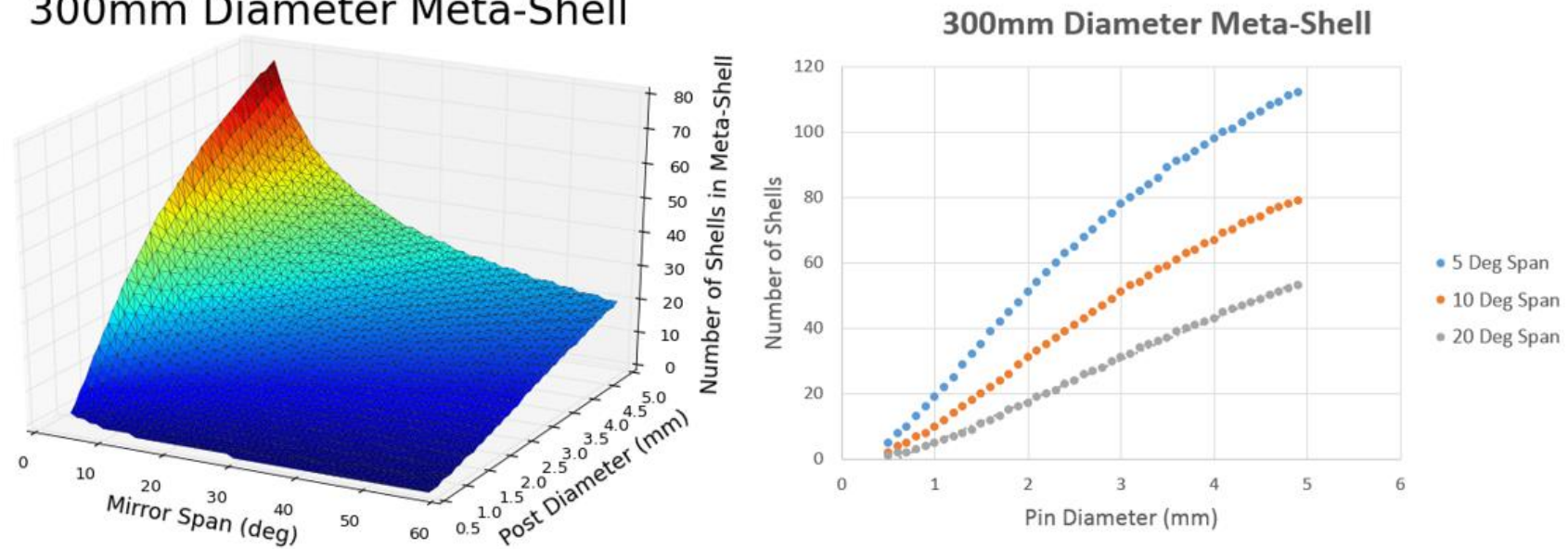

Figure 6. Feasible designs with positive stress margins are below the surface (left). Total bond area can be increased by decreasing the mirror span or increasing the post diameter (right).

The three parameters must be traded against each other to develop an optimal design considering bond strength, mirror effective area, and manufacturability. Increasing the bond size increases the bond strength but blocks incoming $\mathrm{x}$-rays, reducing the effective area. Decreasing the mirror span increases the bond strength but results in more mirror segments that must be fabricated and assembled into the meta-shell. Decreasing the number of layers per meta-shell increases the bond strength but reduces the effective area requiring additional meta-shells to be fabricated. An example of a feasible $1.3 \mathrm{~m}$ mirror assembly design using $0.4 \mathrm{~mm}$ thick silicon mirror segments is shown in Table 1 . For this design, the azimuthal span, determined by degree of segmentation of the annulus, was selected to yield a mirror segment chord width approximately equal to the $100 \mathrm{~mm}$ axial length of segments. The approximately square length-to-width aspect ratio is convenient for fabrication, metrology, and mounting. The bond diameter was selected to be $4 \mathrm{~mm}$, which blocks $9 \%$ of the mirror area. The feasibility of the design, accuracy of the mathematical model, and assumption that bond strength is the limiting factor were verified using a detailed Finite Element Model (FEM) of the meta-shells shown in Figure 8.

Table 1. Example of a $1.3 \mathrm{~m}$ diameter, $5 \mathrm{~m}$ focal length mirror assembly made from six meta-shells

\begin{tabular}{|c|c|c|c|c|c|c|}
\hline $\begin{array}{c}\text { Meta-shell } \\
\text { number }\end{array}$ & $\begin{array}{c}\text { Structural shell } \\
\text { thickness } \\
(\mathbf{m m})\end{array}$ & $\begin{array}{c}\text { Innermost } \\
\text { mirror } \\
\text { diameter }(\mathbf{m m})\end{array}$ & $\begin{array}{c}\text { Outermost } \\
\text { mirror } \\
\text { diameter (mm) }\end{array}$ & $\begin{array}{c}\text { Layers in } \\
\text { meta-shell }\end{array}$ & $\begin{array}{c}\text { Mirror } \\
\text { segments in } \\
\text { meta-shell }\end{array}$ & $\begin{array}{c}\mathbf{1} \text { keV effective } \\
\text { area }\left(\mathbf{c m}^{2}\right)\end{array}$ \\
\hline $\mathbf{1}$ & 5 & 349 & 455 & 24 & 768 & 211 \\
\hline $\mathbf{2}$ & 7 & 480 & 597 & 23 & 920 & 338 \\
\hline $\mathbf{3}$ & 9 & 625 & 752 & 22 & 1056 & 487 \\
\hline $\mathbf{4}$ & 11 & 791 & 922 & 20 & 1200 & 616 \\
\hline $\mathbf{5}$ & 13 & 973 & 1106 & 18 & 1296 & 722 \\
\hline $\mathbf{6}$ & 15 & 1164 & 1296 & 16 & 1344 & 782 \\
\hline
\end{tabular}




\subsection{Structural shell thickness}

The required thickness of the silicon structural shell is determined in two ways. First, the stress in the structural shell must be low. Figure 7 shows a plot of structural thickness versus maximum stress. Second, the flexibility of the structural shell should have a minimal impact on the bond strength. Put another way, the structural shell should be well approximated by an infinitely rigid constraint relative to the mirror segment and mounting post stiffness. In the case of the innermost meta-shell of the design shown in Table 1, a thickness of $5 \mathrm{~mm}$ satisfies both of these criteria. Analysis shows the structural shell thickness should increase approximately linearly with diameter, as with the Chandra full shell design [2].
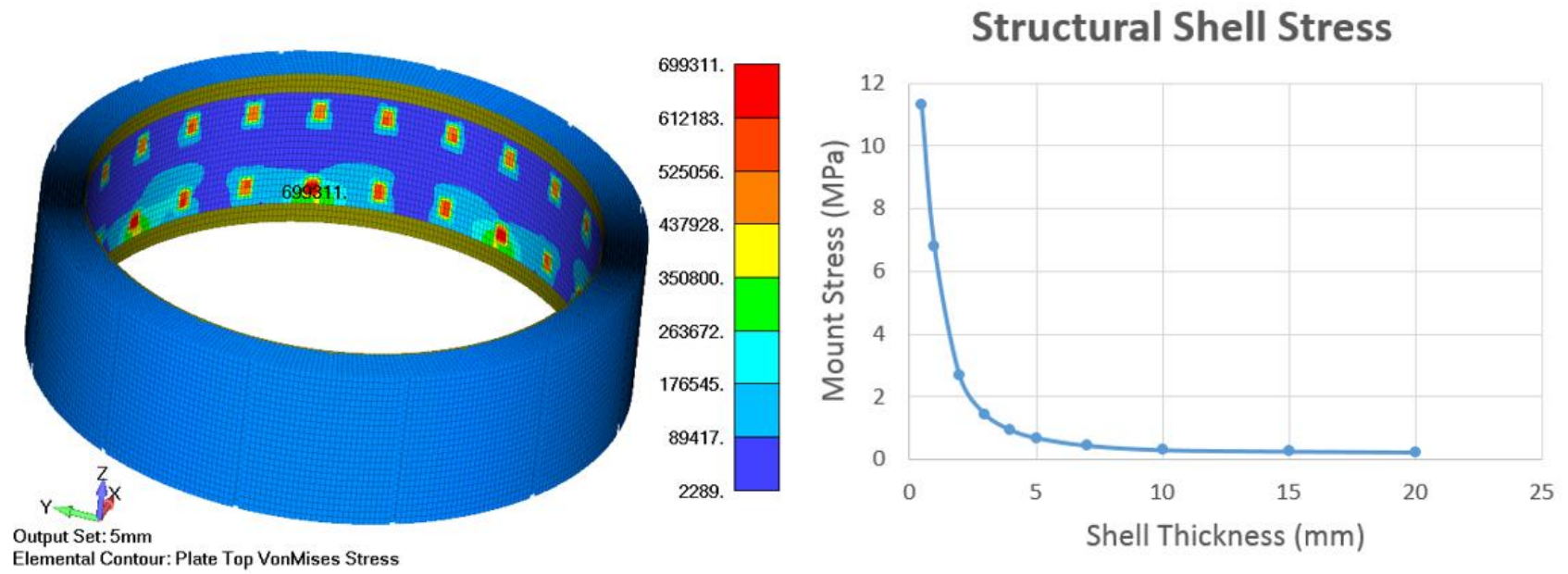

Figure 7. Stress plot of a structural shell (left). The stress decreases as the shell thickness increases (right).

\subsection{Opto-mechanical analysis}

A high fidelity FEM of a meta-shell was generated using a custom software tool that reads in the optical prescription and outputs a NASTRAN compatible FEM with hundreds of mirror shells accurately represented. This model was used to perform opto-mechanical analyses wherein the distortions determined by FEA are ray-traced to predict the resulting xray performance. This method was used to verify the gravity distortions are sufficiently small to support ground based $\mathrm{x}$-ray testing and determine the sensitivity to thermal gradients pending the completion of a full Structural Thermal Optical Performance (STOP) analysis. Table 2 summarizes the opto-mechanical analysis results. Figure 8 shows the distortion and performance of a meta-shell subjected to an axial gravity load.

Table 2. Summary of opto-mechanical analysis results.

\begin{tabular}{|c|c|c|}
\hline Load Case & HPD (arc-sec) & RMSD (arc-sec) \\
\hline $1 \mathrm{~g}$ axial & 0.2 & 0.4 \\
\hline $1 \mathrm{~g}$ lateral & 1.9 & 5.2 \\
\hline $1^{\circ} \mathrm{C}$ bulk temperature change & 0.3 & 0.4 \\
\hline $1^{\circ} \mathrm{C}$ axial temperature gradient & 0.1 & 0.1 \\
\hline $1^{\circ} \mathrm{C}$ lateral temperature gradient & 0.3 & 0.8 \\
\hline $1^{\circ} \mathrm{C}$ radial temperature gradient & 0.3 & 0.4 \\
\hline
\end{tabular}



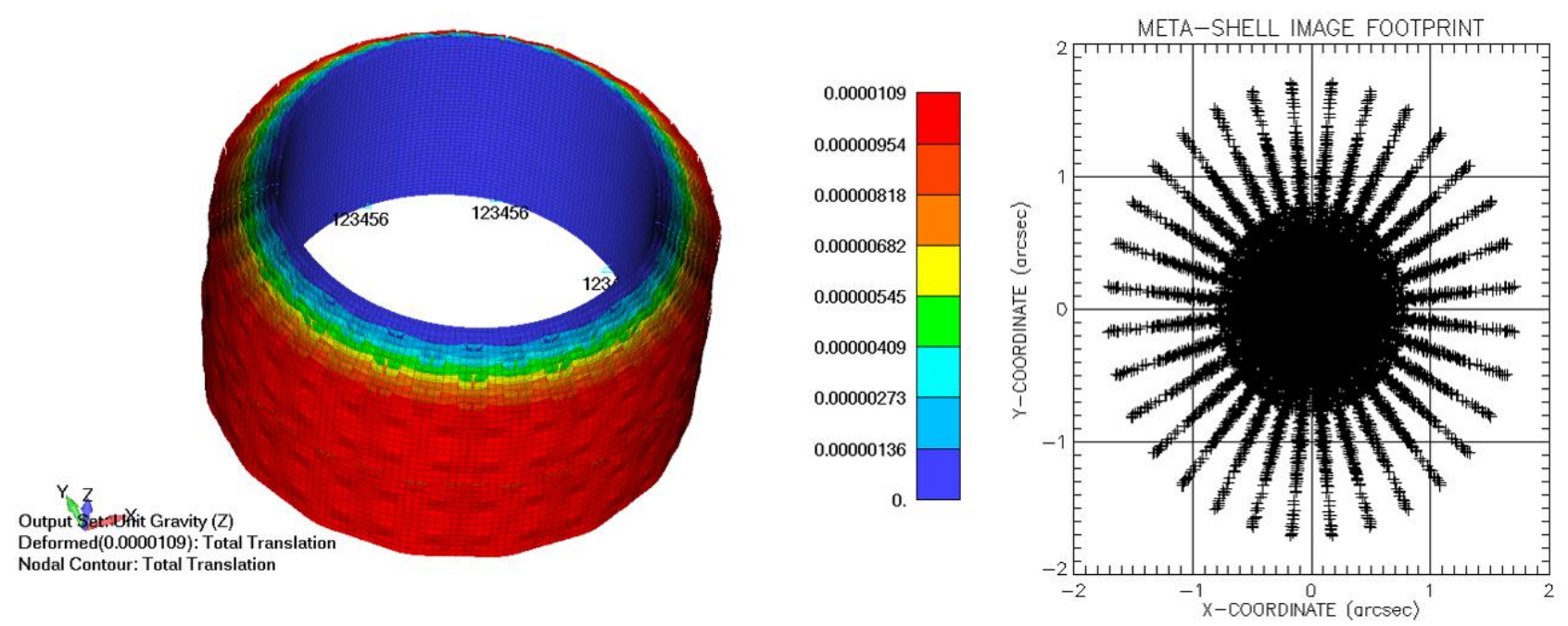

Figure 8. Deformation of a meta-shell under axial gravity load (left) and predicted x-ray image (right).

\section{CONCLUSIONS}

The meta-shell approach combines the fabrication advantages of segmented x-ray optics with the integration advantages of full shell optics. A meta-shell construction method using a four point semi-kinematic mount to layer interlocking mirror segments onto a central structural shell has been developed. Initial testing shows the distortion of the four point mount is minimal. Extensive analysis was performed to demonstrate the feasibility of the four point mount and metashell approach. A mathematical model was developed to aid in the selection of meta-shell design parameters and verify the assembly can accommodate launch loads. A detailed FEM of a meta-shell was used to verify the mathematical model and perform opto-mechanical analysis predicting the $\mathrm{x}$-ray performance under various mechanical and thermal loads. 


\section{REFERENCES}

[1] Bookbinder, J. A., Smith R. K., Hornschemeier, A., et al, “The Constellation-X Observatory,” Proc. SPIE 7011, (2008).

[2] Cohen, L. M., et al., "Structural considerations for fabrication and mounting of the AXAF HRMA optics," Proc. SPIE $1303,(1990)$.

[3] Weisskopf, M. C., et al., "Beyond Chandra - the X-ray Surveyor," Proc. SPIE 9510, (2015).

[4] McClelland, R. S., et al., "Design and analysis of the International X-Ray Observatory mirror modules," Proc. SPIE 7732, (2010).

[5] Collon, M. J., et al., "Silicon Pore Optics development for ATHENA," Proc. SPIE 9603, (2015).

[6] Craig, W. W., et al., "Fabrication of the NuSTAR flight optics," Proc. SPIE 8147, (2011).

[7] McClelland, R. S., et al., "Design and analysis of modules for segmented X-ray optics," Proc. SPIE 8443, (2012)

[8] Gondoin, P., et al., "X-ray spectroscopy mission (XMM) telescope development,” Proc. SPIE 2209, (1994)

[9] Waldman, M., "The alignment test system for AXAF-I's High Resolution Minor Assembly,” Proc. SPIE 2515, (1995)

[10] Choi, M. K., "Thermal Considerations for Meeting $20^{\circ} \mathrm{C}$ and Stringent Temperature Gradient Requirements of IXO SXT Mirror Modules," Proc. SAE 2009-01-2391, (2009) 